\title{
Evaluation of the Quality of Hospital-Based Management of Severe Acute Malnutrition in Children under 5 in Benin
}

\author{
Tchasso Serge Kenao ${ }^{1}$, Charles Sossa Jerome ${ }^{1}$, Yolaine Glèlè Ahanhanzo ${ }^{2}$, \\ Ghislain Emmanuel Sopoh ${ }^{3}$, Patrick Makoutodé4, Victoire Damienne Agueh ${ }^{1}$
}

\begin{abstract}
${ }^{1}$ Département de Promotion de la Santé, Institut Régional de Santé Publique, Université d’Abomey-Calavi, Abomey Calavi, Bénin ${ }^{2}$ Département d’Épidémiologie et de Biostatistique, Institut Régional de Santé Publique, Université d'Abomey-Calavi, Abomey Calavi, Bénin

${ }^{3}$ Département de Santé et Environnement, Institut Régional de Santé Publique, Université d’Abomey-Calavi, Abomey Calavi, Bénin ${ }^{4}$ Département de Politique et Système de Santé, Institut Régional de Santé Publique, Université d’Abomey-Calavi, Abomey Calavi, Bénin

Email: tkenao@yahoo.com
\end{abstract}

How to cite this paper: Kenao, T.S., Sossa Jerome, C., Glèlè Ahanhanzo, Y., Sopoh, G.E., Makoutodé, P. and Agueh, V.D. (2017) Evaluation of the Quality of Hospital-Based Management of Severe Acute Malnutrition in Children under 5 in Benin. Food and Nutrition Sciences, 8, 1012-1021. https://doi.org/10.4236/fns.2017.811073

Received: October 12, 2017

Accepted: November 20, 2017

Published: November 23, 2017

Copyright $\odot 2017$ by authors and Scientific Research Publishing Inc. This work is licensed under the Creative Commons Attribution International License (CC BY 4.0).

http://creativecommons.org/licenses/by/4.0/

\begin{abstract}
Introduction: The aim of the study was to assess the quality of the management of severe acute malnutrition in the Mono Departmental Hospital Center (CHD) in Benin. Methods: This was a cross-sectional and evaluative study which took place from 03 February to 20 July 2016. The study was carried out in the mono departmental hospital of Lokossa. The non-probabilistic sampling method was used to select all our study materials and all our targets were systematically selected. Our study materials involved observation checklist, guidelines documents and questionnaires to collect data. The assessment of the quality of the hospital management of severe acute malnutrition was based on national and international standards like Benin's national protocol of acute malnutrition management. Results: In our study, 27 cases of severe acute malnutrition (SAM) in children were considered. The median age of those children was 12 months. The rates of the components inputs, process and results were $25 \%$ (poor), 58.33\% (acceptable) and 40\% (poor), respectively. The sub components with respect to the norms were all related to the management of severe acute malnutrition like, availability of therapeutic foods ready for use, availability of management protocol, availability of trained and supervised staff in the management of the severe acute malnutrition and the proportion of dead and cured children. The study showed that the quality of the management of severe acute malnutrition at the mono departmental hospital of Lokossa was poor with a rate of $41.38 \%$. Conclusion: The quality of the management of severe acute malnutrition at the mono departmental hospital of Lokossa was poor. The sub com-
\end{abstract}


ponents that need to improve were the availability of therapeutic foods ready for use, availability of management protocol, along with the training and supervision of staff in charge of the management of severe acute malnutrition.

\section{Keywords}

Evaluation, Severe Acute Malnutrition, Benin

\section{Introduction}

In developing countries, child malnutrition is still one of the main causes of child death and a major public health problem [1]. According to the World Health Organization (WHO), nearly 10 million children suffer from malnutrition. In sub-Saharan Africa, malnutrition had always been affecting children under five years of age and is responsible of about $60 \%$ death of children under five years of age. In Benin, the prevalence of severe acute malnutrition (SAM) is estimated to be $8 \%$ [2]. The frequent elevation malnutrition demands a strong involvement of health systems for its management. Adequate management of severe acute malnutrition in hospitals involves the management of clinical complications and the nutritional recovery [3] [4] [5]. According to the national protocol for the management of acute malnutrition in Benin, management of severe acute malnutrition with complications is performed at hospitals in three phases (stabilization, transition, rehabilitation) considering four important components which are: nutritional, medical, psychosocial and nutritional surveillance in accordance with the WHO guidelines [4] [6].

As a departmental hospital, the skills in care and services in accordance with the "protocol for the management of acute malnutrition in Benin" should ensure effective management of SAM. Adequate interventions in the management of SAM are required in order to reduce related mortality in children under 5 years of age. The purpose of the study was to assess the quality of the management of severe acute malnutrition in the mono Departmental Hospital Center (CHD) in Lokossa.

\section{Methods}

\subsection{Setting}

Our study was conducted at the Mono CHD of Lokossa in pediatric service. The town of Lokossa located in the South-West of Benin is 110 miles from Cotonou. The hospital provides care and services to the department of Mono and Couffo, the population of which is estimated or esteemed to 1.242 .571 inhabitants, with 197.567 children under five years of age according to the 2012 census or enumeration. The prevalence of SAM was respectively $2.2 \%$ and $5 \%$ in the Couffo and Mono departments in 2014 [6]. The dietary habits of the population were mainly based on cereals and tubers [2]. 


\subsection{Study Design and Materials}

This was a cross-sectional and evaluative study which took place from 03 February to 20 July 2016. The study population and material included:

$\checkmark$ children under 5 years of age admitted to the Mono departmental hospital for severe acute malnutrition;

$\checkmark$ health professionals involved in the management of SAM in the pediatric ward;

$\checkmark$ mothers of children with SAM admitted in the hospital during the period of 1 to 30 June 2016;

$\checkmark$ medical records of children under 5 admitted to CHD Mono for severe acute malnutrition.

\subsection{Inclusion and Exclusion Criteria}

\section{* Inclusion criteria}

Following subjects were included in the study:

$\checkmark$ children under 5 years of age admitted to the Mono departmental hospital for severe acute malnutrition;

$\checkmark$ health professionals involved in the management of SAM in the pediatric ward;

$\checkmark$ mothers of children with SAM admitted in the hospital during the period of 1 to 30 June 2016.

\section{* Exclusion criteria}

Health professionals involved in the management of SAM in the pediatric service for less than one month were excluded from the study.

\section{Sampling Method}

The non-probabilistic sampling method was used to identify participants and material that were systematically selected.

The sample of participants included 6 health professionals of the pediatric service, 27 children with SAM and 8 mothers of children with SAM within the period from 1st till 30 June 2016.

\subsection{Study Variables}

Figure 1 below describes the frame work of the management of SAM. The three components: inputs, process and results were used to explain the quality of management of SAM in children under five years.

\subsection{Measurement of Variables}

For the operational aspects of the variables, we adapted our rating scale and appreciation to the one proposed by Myriam Hubinon [7]. Predetermined scores were awarded to variables according to the criteria met for their appreciation. Those scores varied between 1 (if the criteria was met), and 0 (if the criteria was not met) for the variable with only one assessment criteria. 


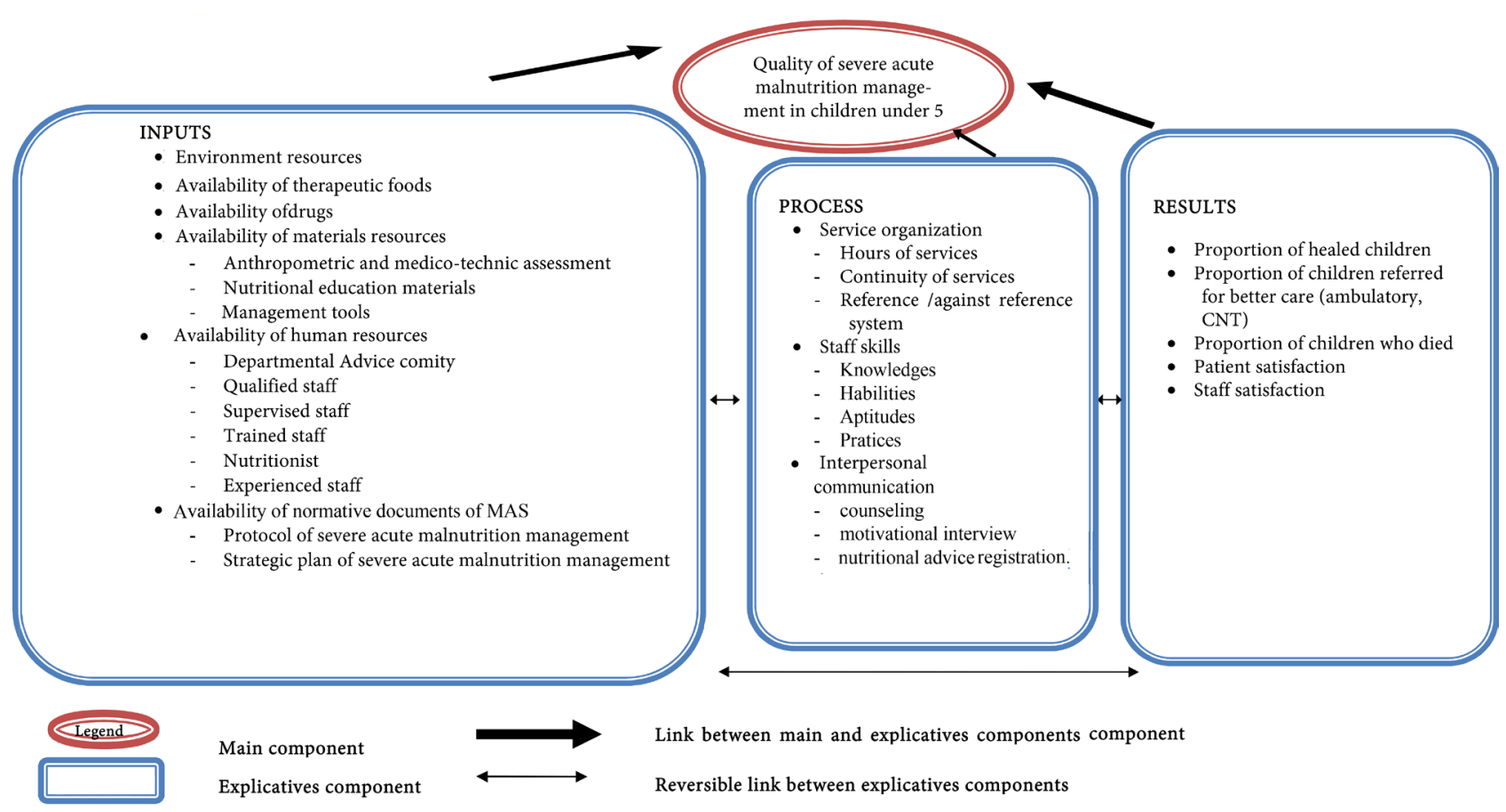

Figure 1. Conceptual framework of severe acute malnutrition management at the Mono departmental hospital of Lokossa in Benin, adapted from Donabedian model.

But for variables with more than one assessment criteria, the score awarded to the variable was 1 if more than $75 \%$ of the expected criteria were met and 0 if less than $75 \%$ of expected outcomes were observed.

In accordance with the protocol, the components were qualified as:

- good if the score was more than or equal to $75 \%$ of the score expected,

- bad if the score was less than $75 \%$ of the score expected.

\subsection{Data Collection Procedures}

The means and tools used to collect data were; interview from the head of the pediatric department and questionnaires for health professionals based on their qualification, training and knowledge in the management of SAM.

Direct observation was used to assess the working environment (medical and technical equipment, the availability of therapeutic foods, medicines, technical support, and health professional skills) and information about anthropometric data and treatment were assessed from the reports and medical records or files of children admitted for SAM.

Two investigators were hired and trained about data collection sheets and tools as well as how to employ the method of work. Data collection was supervised by a student at the end of the training of Master's Program in Nutrition.

\subsection{Ethical Considerations}

The approval of the hospital was obtained prior to any action. An oral consent was obtained from all the targets before any data collection. That consent claimed that targets may withdraw their participation in the study at any time 
without any risk. Anonymity and confidentiality of the information were also ensured.

\section{Results}

\subsection{Characteristics of Children}

Table 1 summarizes the characteristics of children; a total of 27 children with severe acute malnutrition (SAM) participated in the study. The median age of children was 12 months ( 4 - 20 months). The sex ratio was 0.5 . Among 27 children, only 3 with a complete treatment with all the nutritional, medical, psychosocial and nutritional surveillance support were included.

\subsection{Appreciation of the Component «Inputs» of Management of Severe acute Malnutrition}

Table 2 below describes the appreciation of the component "inputs". The "inputs" obtained 3 points out of 12 , being $25 \%$. Consequently, the assessment of this component showed that the availability and functionality of inputs for the management of severe acute at the Mono CHD in Lokossa were poor. The sub-components of the no-compliant inputs were: environmental resources, therapeutic foods, nutritional education materials, departmental advice comity, qualification of staff, staff training, supervision of staff and availability of normative documents.

\subsection{Appreciation of the Component «Process» of Management of Severe Acute Malnutrition}

Table 3 below describes the appreciation of the component "Process". In our study, the component «Process» had a score of 7 out of 12 required, making a quality score of $58.33 \%$.

\subsection{Appreciation of the Component «Results» of Management of Severe acute Malnutrition}

Table 4 below summarizes the appreciation of the component "Results". The "results" component obtained 2 points out of 5, being 40\% (under 75\%). The

Table 1. Socio-demographic characteristics and health aspects children with MAS Mono department, Lokossa in $2015(\mathrm{n}=27)$.

\begin{tabular}{ccc}
\hline Variables & Effective & $\%$ \\
\hline Sex & 9 & 33.33 \\
\hline Female & 18 & 66.66 \\
Male & 3 & 11.11 \\
\hline Admission diagnosis & 2 & 7.40 \\
\hline $\begin{array}{c}\text { Severe acute malnutrition } \\
\text { Marasmus }\end{array}$ & 22 & 81.48 \\
\hline
\end{tabular}


Table 2. Score of the component «Inputs» of management of severe acute malnutrition.

\begin{tabular}{cccc}
\hline Variables & Observed score & Score expected & Frequency (\%) \\
\hline Environment resources & 0 & 1 & $25^{*}$ \\
Therapeutic foods & 0 & 1 & 0 \\
Essential drugs & 1 & 1 & 100 \\
$\begin{array}{c}\text { Anthropometric and } \\
\text { medico-technic assessment }\end{array}$ & 1 & 1 & $80^{* *}$ \\
Nutritional education materials & 0 & 1 & 0 \\
Management tools & 1 & 1 & $80^{* *}$ \\
Departmental advice comity & 0 & 1 & 0 \\
Qualified staff & 0 & 1 & $50^{* * *}$ \\
Trained staff & 0 & 1 & 0 \\
Nutritionist & 0 & 1 & 0 \\
Supervised staff & 0 & 1 & 0 \\
Normative documents of MAS & 0 & 1 & 0 \\
Total & 3 & 12 & 25 \\
\hline
\end{tabular}

*: One modality (lighting) on 4 (lighting, propriety, ventilation, privacy) was wright; $25 \%<75 \%$; so score was " 0 "; **: 4 on 5 anthropometric material were availability; $80 \%>75 \%$ so the score is " 1 "; *** 6/12 health professionals were qualified; $50 \%<75 \%$ so the score is " 1 ".

Table 3. Score of the component «Process» of management of severe acute malnutrition.

\begin{tabular}{|c|c|c|c|}
\hline Variables & Observed score & Score expected & Frequency (\%) \\
\hline \multicolumn{4}{|l|}{ Service organization } \\
\hline Hours of services & 1 & 1 & $83^{*}$ \\
\hline Continuity of services & 1 & 1 & 100 \\
\hline $\mathrm{RCR}^{1}$ system & 1 & 1 & 100 \\
\hline Hosting & 1 & 1 & $86^{\star *}$ \\
\hline \multicolumn{4}{|l|}{ Staff skills } \\
\hline Knowledge & 0 & 1 & 43 \\
\hline Abilities & 1 & 1 & $81^{* * *}$ \\
\hline Attitudes & 1 & 1 & $87^{* * * *}$ \\
\hline Aptitudes & 0 & 1 & 0 \\
\hline Practices & 0 & 1 & 60 \\
\hline \multicolumn{4}{|l|}{ Interpersonal communication } \\
\hline counseling & 0 & 1 & 0 \\
\hline Motivational interview & 0 & 1 & 0 \\
\hline Nutritional advice registration & 1 & 1 & 100 \\
\hline Total & 7 & 12 & 58.33 \\
\hline
\end{tabular}

*: 10 of 12 health professional were on time; that is $83 \%>75 \%$ so the score is 1 point; ${ }^{* *}$ : 6 steps on 7 were respected in hosting process (acronym BERCER); that is $86 \%>75 \%$ so the score is 1 point; ${ }^{\star * *}$ : All the steps were not effective; that explain the point of score (1 point); ${ }^{1}$ : Reference_Contre_Reference. 
Table 4. Score of the component «Results» of management of severe acute malnutrition.

\begin{tabular}{lccc}
\hline \multicolumn{1}{c}{ Variables } & Observed score & Score expected & Frequency (\%) \\
\hline Proportion of healed children & 0 & 1 & $25.92^{*}$ \\
Proportion of children referred for better care & 1 & 1 & 100 \\
Proportion of children who died & 0 & 1 & $22.22^{* *}$ \\
Patient satisfaction & 1 & 1 & $88^{* * *}$ \\
Staff satisfaction & 0 & 1 & 0 \\
Total & 2 & 5 & 40
\end{tabular}

*:7 out of 27 children were healed; that was $25.92 \%<75 \%$ so the score is 0 point; ${ }^{* *}$ : 6out of 27 children were died; $22.22 \%<75 \%$ so the score is 0 point; ${ }^{\star * *}$ : Only one client out of 8 in the focus group was not satisfied; $88 \%>75 \%$ that explain the point of the score.

appreciation of the component was rated poor. The scores for this component are summarized in the Table 4.

\subsection{Overall Appreciation of Management of Severe Acute Malnutrition}

Table 5 below summarizes the appreciation of the overall quality of management of severe acute malnutrition.

\section{Discussion}

To assess the quality of the management of SAM in children in Mono departmental hospital, the components inputs, process and results were rated. The final score showed that the quality of the management of severe acute malnutrition was poor.

\subsection{Characteristics of Severe Acute Malnutrition Children}

Similar studies of Savadogo et al. [8] and Singh et al. showed that among the children included in the study, the male gender was much more affected with a proportion of $54.6 \%$ in the study by Singh et al. [9]. This difference could be explained by the size of the different samples. The size of the sample from Burkina and India was 1322 and 1229, respectively, compared to 28 cases in our study.

\subsection{Inputs of Management of Severe Acute Malnutrition}

Bambara showed in his study on nutritional surveillance that $79.07 \%$ of health professionals had been supervised in the management of SAM [10]. This difference may be explained by the fact that the study was carried out in health units of the health district which make plans and carry out combined or integrated monitoring in each quarter. Such monitoring will lead to key recommendations in a way to improve the SAM management. Also the availability and functionality of a good reference and against the reference system is a major asset for the success of SAM management even with complications. 
Table 5. Appreciation of quality of management of severe acute malnutrition.

\begin{tabular}{ccc}
\hline Components & Quality score (\%) & Appreciation \\
\hline Inputs & 25 & Poor \\
Process & 58.33 & Poor \\
Results & 40 & Poor \\
Total & 41.38 & Poor \\
\hline
\end{tabular}

Quality of the management of severe acute malnutrition at the mono departmental hospital was poor $41.38 \%$ (under $75 \%$ ).

\subsection{Process of Management of Severe Acute Malnutrition}

The results concerning knowledge and skills in the SAM management are different from those obtained by Testa et al. in their study [11]. In their study, 2 out of 19 health professionals were skilled and aware of the 3 steps and 4 components of SAM treatment. This difference could be explained by the fact that their study was carried out in 19 centers. In those 19 centers, 7 were handled by the midwives and 12 centers by social welfare staff taking care of cases of Moderate Acute Malnutrition in the communities. The capacities of health professional of pediatric ward should be strengthened for effective management of the SAM in the hospital.

The results obtained from the practical assistance of health professional were different from those obtained by Aouehougon et al. in their study conducted in the health district of Tougan [12]. In 2007, she found in Tougan, that nutritional index was specified in $70 \%$ of cases and tracking curve drawn and followed up in $60 \%$ of cases. This difference could be explained by the sample size. In our study, only the head of the pediatric ward or service was the subject of direct observation in order to assess practical assistance during the treatment of SAM in children. The score of that sub component was found to be poor.

\subsection{Results of Management of Severe Acute Malnutrition}

The proportion of healed children was different from the one obtained by Hossain et al. in 2016, where they $88 \%$ of children cured from SAM [13]. This difference could be explained by the sample size, which was 138 children in their study compared to 27 in our study. Our result was also different from the one observed by Nguefack et al. in Cameroon. They found that (75.6\%) 31 children out of 41 being properly treated for SAM [14]. This difference could be explained by the use of therapeutic milk as a treatment to manage SAM after complications had been handled. Their study was also a clinical trial compared to our study which is just an observational study.

The proportion of children who died was different from the results obtained by Hossain M. et al. and Nguefack et al. Their results were $10.19 \%$ and $21.9 \%$ respectively. These differences could be explained by their respective sizes of the samples which were 138 and 41 participants. In the study of Savadogo et al., 16\% children were observed who died [8]. This study was carried out in urban areas 
and aimed to assess the nutritional status and causes of death of children admitted to a nutritional rehabilitation center, in order to improve the management of those centers. The difference met could also be explained with those issues.

The result concerning the satisfaction of mothers was different from the one found by Kiemde et al. in their study in Banfora, Burkina Faso, showing 32\% of non-satisfaction. The main reason mentioned was the use of the same pant to assess the weight of SAM children. This difference could be explained by the fact that in our study, a scale with the double weighing was used to assess children's weight [15]. In the study of Testa et al., 39\% of mothers were observed to be satisfied. The difference could be explained by the sample size used to assess the satisfaction rate.

\subsection{Quality of Management of Severe Acute Malnutrition}

In a study, Bitwe et al. [16] found that the quality of the management of children in hospitals was poor. However, the difference between the components used to assess the quality of the management could be explained by the fact that this study had a global aim to assess all childhood illnesses compared to our study.

One of the limitations of this study is that since the study took place in only one hospital, so the results are not applicable for all the hospitals in the country. Variables were scored between 0 and 1. Despite these limitations, the study brought useful results to improve the SAM management in hospitals.

\section{Conclusion}

The quality of the management of severe acute malnutrition at the mono departmental hospital of Lokossa in Benin was observed to be poor. The sub components that need to improve were availability of therapeutic foods ready for use, availability of management protocol, the training and supervision of staff in charge of the management of severe acute malnutrition and best practices of health professional. Specific and sensible interventions are required to avoid severe and irreversible consequences of malnutrition in children.

\section{References}

[1] Black, R.E., Victora, C.G. and Walker, S.P. (2013) Maternal and Child under Nutrition and Overweight in Low-Income and Middle-Income Countries. Lancet, 382, 427-451. https://doi.org/10.1016/S0140-6736(13)60937-X

[2] Institut National de la Statistique et de l'Analyse Économique (INSAE) et ICF International: [Demographic and Health Survey Report and Multiple Indicators.] Benin, EDS-MICS-IV.2011-2012, Cotonou, 42 p.

[3] ArenasMoya, D., Plascencia Gaitan, A., Ornelas Camacho, D. and Arenas Marquez, H. (2016) Hospital Malnutrition Related to Fasting and Underfeeding: Is It an Ethical Issue? Nutrition in Clinical Practice, 31, 316-324. https://doi.org/10.1177/0884533616644182

[4] Ministère de la Santé (2011) Protocole nationale de prise en charge de la malnutrition aigüe: Bénin. [National Protocol of Acute Malnutrition Management in Benin.] Cotonou, 193 p. http://www.sante.gouv.bj/ 
[5] Ashworth, A., Khanum, S., Jackson, A. and Schofield, C. (2003) Guidelines for the Inpatient Treatment of Severely Malnourished Children. South-East Asian Regional Office, World Health Organization, New Delhi, 51 p. (SEARO Technical Publication no. 24)

[6] Organisation Mondiale de la Santé (2015) Lignes directrices: mises à jour de la prise en charge de la malnutrition aiguë sévère chez le nourrisson et chez l'enfant. [Guidelines Updates of Severe Acute Malnutrition Management in Infants and Children.] OMS, WHO, Genève, 128 p.

[7] Hubinon, M. (2010) Management des unités de soins, de l'analyse systémique à l'évaluation de la qualité. [Care Unit's Management, Systemic Analysis to Quality Assessment.] Breckuniv, Bruxelles, Belgique, 395 p.

[8] Savadogo, L., Zoetaba, I., Donnen, P., Hennart, P., Sondo, B.K., and Dramaix, M. (2007) Management of Severe Acute Malnutrition in an Urban Nutritional Rehabilitation Center in Burkina Faso. Revue d'Épidémiologie et de Santé Publique, 55, 265-274. https://doi.org/10.1016/j.respe.2007.05.006

[9] Singh, K. (2013) Management of Children with Severe Acute Malnutrition: Experience of Nutrition Rehabilitation Centers in Uttar Pradesh, India. Accompanying Editorials, 51, 17-20.

[10] Bambara, R. (2011) Déterminants de la faible qualité de la surveillance nutritionnelle des enfants de moins de cinq ans dans le district sanitaire de Koupéla, région du Centre Ouest du Burkina Faso. [Determinants of the Poor Quality of $\mathrm{Nu}$ tritional Surveillance of Children under Five Years of Age in the Health District of Koupela, West Central Region of Burkina Faso.] https://www.memoireonline.com/

[11] Testa, J., Sinnaeve, O., Ybrahim, Y. and Ayivi, B. (2008) Evaluation de la qualité du dépistage et de la prise en charge de la malnutrition infantile à Cotonou. [Evaluation of the Quality of Screening and Management of Malnutrition Child in Cotonou.] Medicina Tropical, 68, 45-50.

[12] Aouehougon, O. (2007) La malnutrition protéino-énergétique et ses facteurs de risque chez les enfants de moins de 5 ans dans le DS de Tougan. [Protein-Energy Malnutrition and Its Risk Factors in Children under 5 Years of Age in Tougan Health District.] 136 p. https://www.memoireonline.com/

[13] Hossain, M., Nina, S., Tahmeed, A., Miah, G.M., Kazi, M., Jamil, B.N., et al. (2009) Severe Acute Malnutrition. Experience in Managing Severe Malnutrition in a Government Tertiary Treatment Facility in Bangladesh. Journal of Health, Population and Nutrition, 27, 72-79. https://doi.org/10.3329/jhpn.v27i1.3319

[14] Nguefack, F., Akazong, C., Keugoung, B., Kamgaing, N. and Dongmo, R. (2015) Prise en charge de la malnutrition aigue sévère chez les enfants avec préparation locales et alternatives aux F-75 et F-100: Résultats et défis; [Management of Severe Acute Malnutrition in Children with Local Preparation and Alternatives to the F-75 and F-100: Results and Challenges.] Pan African Medical Journal, 21, 329. https://doi.org/10.11604/pamj.2015.21.329.6632

[15] Kiemde, S.A. (2005) Etude des facteurs expliquant le faible taux de couverture de la surveillance nutritionnelle des enfants de 0 à 5 ans dans le district sanitaire de Banfora. [Study of the Factors Explaining the Low Coverage Rate of Nutritional Surveillance of Children from 0 to 5 Years Old in Banfora Health District.] Biologie et Médecine, 15. https://www.memoireonline.com

[16] Bitwe, R. (2007) Qualité des soins donnés aux enfants gravement malades dans un hôpital provincial en Afrique Centrale. [Quality of Care for Critically Ill Children in a Provincial Hospital in Central Africa.] Public Health, 19, 401-411. 